\title{
Estado nutricional e dieta enteral prescrita e recebida por pacientes de uma Unidade de Terapia Intensiva
}

\author{
Nutritional status and enteral diet prescribed and received by patients of an \\ intensive care unit
}

Maria Denise Stefanello', Fabiana Assmann Poll ${ }^{1}$

${ }^{1}$ Curso de Nutrição, Universidade de Santa Cruz do Sul (UNISC) - Santa Cruz do Sul (RS), Brasil.

DOI: http://dx.doi.org/10.7322/abcshs.v39i2.625

\begin{abstract}
RESUMO
Introdução: A nutrição enteral representa uma possibilidade terapêutica de manutenção ou recuperação do estado nutricional, para tanto, tão essencial quanto a prescrição da terapia adequada às necessidades do paciente é a constatação de que ele receberá o que lhe é prescrito. Objetivo: Verificar o estado nutricional de pacientes em uso da terapia de nutrição enteral, relacionando com a adequação do valor energético, proteico e volume prescrito e recebido durante internação na Unidade de Terapia Intensiva de um hospital de Santa Cruz do Sul, Rio Grande do Sul. Métodos: Estudo observacional descritivo retrospectivo e quantitativo. Foram avaliados pacientes adultos internados na Unidade de Tratamento Intensivo em uso de terapia de nutrição enteral exclusiva por mais de 72 horas, de julho a setembro de 2011. Considerou-se satisfatória a adequação $\geq 60 \%$ para calorias e proteínas relacionando o recebido e o prescrito da dieta enteral e $\geq 90 \%$ para a adequação do volume de dieta. Para análise do estado nutricional foi utilizado o índice de massa corporal a partir de uma amostra não probabilística de caráter intencional. Resultados: Totalizou-se 36 pacientes. Desses, 16,7\% estavam em magreza e $44,44 \%$ em eutrofia. Aproximadamente 44,4 e $22,2 \%$ dos pacientes, respectivamente, atingiram $\geq 60 \%$ das calorias e proteínas prescritas, destacando-se aqueles em estado nutricional de magreza $(p=0,012)$. Do total de pacientes, $36 \%$ alcançaram a adequação de volume sem diferença estatisticamente relevante com o estado nutricional $(p=0,771)$. Conclusão: Verificouse que a maioria dos pacientes estava em estado nutricional de eutrofia ou sobrepeso. Adultos em estado crítico receberam menos dieta enteral do que o prescrito.
\end{abstract}

Palavra-chave: estado nutricional; cuidados críticos; nutrição parenteral.

\begin{abstract}
Introduction: Enteral nutrition represents a therapeutic possibility of maintenance or recovery of nutritional status. Therefore, as essential as the proper therapy prescription to the patient needs is the finding that he will receive what is prescribed. Objective: To verify the nutritional status of patients in use of enteral nutrition therapy, relating it to the adequacy of energy and protein value, and the volume prescribed and received during hospitalization in intensive care unit in a hospital in Santa Cruz do Sul, Rio Grande do Sul, Brazil. Methods: Quantitative retrospective descriptive observational study. We evaluated adult patients hospitalized in intensive care unit having an exclusive enteral nutrition therapy for more than 72 hours, from July to September 2011. It was considered satisfactory the adequacy $\geq 60 \%$ for calories and proteins correlating with the receiving and prescribing enteral diet, and $\geq 90 \%$ for the adequacy of the diet volume. For analysis of nutritional status, it was used the body mass index from a nonprobability sample of intentional character. Results: Thirty-six patients were analyzed. Of these, $16.7 \%$ was underweight and $44.44 \%$ was eutrophic. Approximately 44.4 and $22.2 \%$ of these patients reached $\geq 60 \%$ of prescribed calories and proteins, respectively, standing out those who were in an underweight nutritional status $(p=0.012)$. Of the total patients, $36 \%$ reached the adequacy of volume without statistically significant difference from the nutritional status $(p=0.771)$. Conclusion: It was found that most patients were in eutrophic nutritional status or overweight. Adults in critical condition received less enteral diet than prescribed.
\end{abstract}

Keywords: nutritional status; critical care; parenteral nutrition. 


\section{INTRODUÇÃO}

A resolução RDC no 63, da Agência Nacional de Vigilância Sanitária do Ministério da Saúde (ANVISA) ${ }^{1}$, de 06/07/2000, define nutrição enteral (NE) como: alimento para fins especiais, com ingestão controlada de nutrientes, na forma isolada ou combinada, de composição definida ou estimada, especialmente formulada e elaborada para uso por sondas ou via oral, industrializada ou não, utilizada exclusiva ou parcialmente para substituir ou completar a alimentação oral em pacientes desnutridos ou não, conforme suas necessidades nutricionais, em regime hospitalar, ambulatorial ou domiciliar, visando à síntese ou manutenção dos tecidos, órgãos ou sistemas.

Existem no mercado fórmulas enterais industrializadas, as mais utilizadas nos hospitais atualmente, que satisfazem as exigências preconizadas pelo Ministério da Saúde, adequando-se de forma diferenciada a indivíduos de todas as faixas etárias e situações clínicas que necessitem de suporte nutricional. O desafio para os profissionais que atuam na Unidade de Tratamento Intensivo (UTI) continua sendo determinar as necessidades nutricionais que se ajustem a cada paciente ${ }^{2}$.

A maioria dos profissionais concorda que a nutrição enteral apresenta menos riscos e mais vantagens do que a nutrição parenteral, apesar das controvérsias geradas por conclusões baseadas em modelos animais que não se fundamentaram em observações clínicas em seres humanos ${ }^{3}$.

A nutrição enteral surge como uma possibilidade terapêutica de manutenção ou recuperação do estado nutricional e tem importante função fisiológica. A manutenção da morfologia e função do trato gastrointestinal requer a presença intraluminar de nutrientes específicos, vitais para o trofismo e função da parede intestinal. Nos pacientes muito graves, alterações na permeabilidade da parede intestinal podem levar à translocação bacteriana e, consequentemente, à persistência de um quadro séptico. Pacientes graves em nutrição enteral apresentam índices de infecção menores. O tecido linfoide intestinal funciona como um braço aferente imunológico. Há evidências cada vez maiores de que a preservação da massa tecidual linfóide do intestino preserva a imunidade local e sistêmica ${ }^{4}$. Ressalte-se que cada paciente apresenta um desafio individual e a terapia nutricional deve ser adaptada às evoluções ou complicações imprevisíveis ${ }^{3}$.

Na UTI o paciente está predisposto ao catabolismo que acompanha a mobilização de proteínas para que o organismo produza energia e se recupere de suas lesões. Essa resposta orgânica ao estresse, chamada de resposta de fase aguda, expõe o paciente a riscos nutricionais ${ }^{5}$

A equipe multiprofissional de terapia nutricional (EMTN) deve decidir se o objetivo da nutrição enteral é a manutenção ou a reposição nutricional para determinar com acerto o aporte de calorias e nutrientes a serem fornecidos e através de quais formulações, já que a nutrição enteral, como o suporte nutricional de um modo geral, deve proporcionar a redução da velocidade da perda de peso e proteólise e promover o anabolismo ${ }^{6}$.
Consensualmente, acredita-se que quanto antes se inicie o suporte nutricional, melhor, principalmente naqueles pacientes previamente desnutridos ou naqueles para os quais se prevê uma desnutrição rápida e importante ${ }^{4}$.

Tão importante quanto a prescrição da terapia nutricional enteral adequada às necessidades do paciente é a constatação de que o paciente receberá o que lhe é prescrito. O conhecimento dos fatores que impedem a efetiva administração da terapia de nutrição enteral permite a adoção de medidas visando o aporte calórico proteico adequado aos pacientes graves ${ }^{7}$.

No paciente crítico, uma administração de volume menor de dieta enteral do que a prescrição contribui para a desnutrição e suas consequências: aumento da morbimortalidade, tempo de hospitalização e custos com os cuidados à saúde 5 .

Vários fatores podem levar a um suporte nutricional inadequado: intolerância individual do paciente à dieta, sub ou superestimação das necessidades diárias, introdução tardia da terapia de nutrição enteral e também as interrupções para procedimentos de rotina da UTI, evidenciando a importância do conceito de controle de qualidade quando se trata de pacientes críticos.

Para tanto, o objetivo do presente estudo foi verificar o estado nutricional de pacientes em uso da terapia de nutrição enteral, relacionando com a adequação do valor energético, proteico e volume prescrito e recebido durante a internação na UTI do Hospital Santa Cruz, em Santa Cruz do Sul, Rio Grande do Sul.

\section{MÉTODOS}

A pesquisa foi aprovada pelo Comitê de Ética em Pesquisa da Universidade de Santa Cruz do Sul (UNISC) sob o protocolo n $2864 / 11$.

Caracteriza-se por um estudo observacional descritivo retrospectivo de caráter quantitativo de um grupo de pacientes adultos internados em uma UTI de um hospital de Santa Cruz do Sul, Rio Grande do Sul.

A coleta de dados foi realizada a partir de dados secundários das Fichas de Avaliação Nutricional e Acompanhamento do Serviço de Nutrição. Nelas, buscou-se o monitoramento nutricional diário de cada paciente: data de internação na UTI, doença(s), idade, gênero, estatura recumbente ( $\mathrm{em} \mathrm{cm}$ ), peso corporal (em kg) no primeiro atendimento nutricional (obtidos pelas camas balanças disponíveis em todos os leitos da UTI), índice de massa corporal (IMC), fórmula enteral prescrita, valor energético total (VET) diário prescrito e recebido, as proteínas (em g) diárias prescritas e recebidas, volume de dieta diário prescrito e recebido, data de início da terapia de nutrição enteral e número de dias que o paciente se encontrava em nada por via oral (NPO).

Foram incluídos na amostra, não probabilística de caráter intencional, pacientes de ambos os gêneros, adultos, admitidos na UTI com diferentes doenças e necessidades nutricionais, submetidos à terapia de nutrição enteral exclusiva e que a receberam por, no mínimo, 72 horas. Foram excluídos pacientes que receberam 
dieta oral e/ou parenteral concomitante e aqueles que não tiveram aferidas suas medidas antropométricas fundamentais. Observe-se que a UTI dessa instituição é referência na região em atendimento a pacientes vítimas de traumas, portanto, com maior dificuldade para obtenção das medidas antropométricas, o que pode contribuir para uma menor amostra de indivíduos no período estudado.

O método de administração da dieta enteral foi contínuo por bomba de infusão a vácuo com fórmulas industrializadas, em sistema fechado, e avaliadas pela equipe de nutrição para estimativa das necessidades diárias de energia e proteínas de acordo com parâmetros estabelecidos para cada diagnóstico.

Para estimar o valor energético total de cada paciente foi utilizado o valor de $\mathrm{kcal} / \mathrm{kg} /$ dia recomendado pela European Society for Parenteral and Enteral Nutrition (ESPEN) ${ }^{8}$, de 20 a $30 \mathrm{kcal} / \mathrm{kg}$ de peso/dia.

Nos pacientes que não se enquadraram nessas doenças quanto às necessidades proteicas (em $\mathrm{g} / \mathrm{kg} / \mathrm{dia})$, foram utilizadas as recomendações propostas pela National Advisory Group on Standarts and Practice Guidelines for Parenteral Nutrition ${ }^{9,10}$.

Destaca-se que o peso corporal usado para estimar o IMC, o VET e as proteínas foi o peso atual (em $\mathrm{kg}$ ) do paciente.

A adequação energética foi avaliada através do valor calórico prescrito e do ingerido em 24 horas, com base na seguinte fórmula: adequação das calorias (kcal) infundidas $(\%)=\mathrm{kcal}$ infundidas/kcal prescritas x 100.

Para a avaliação da adequação proteica foi usada a fórmula utilizando a quantidade de proteínas (em g) prescrita e recebida nas últimas 24 horas: adequação das proteínas (PTNs) (g) infundidas (\%) = PTNs infundidas/PTNs prescritas $\mathrm{x} 100$.

A adequação do volume foi encontrada comparando o volume de dieta enteral prescrito e recebido nas últimas 24 horas, com base na seguinte fórmula: adequação do volume (em ml) infundido $(\%)$ = volume infundido/volume prescrito $\mathrm{x} 100$.

Foi considerada satisfatória uma adequação $\geq 60 \%$ entre o prescrito e o recebido, tanto para o valor calórico quanto para o proteico, sendo esse valor estabelecido a partir da ideia de que a indicação da terapia nutricional enteral deve ser feita quando a ingestão oral do paciente não prover dois terços a três quartos dos requerimentos nutricionais estipulados para prevenir a desnutrição ${ }^{11}$ e também, tendo em vista que o hospital estruturou recentemente sua EMTN e ainda vem aprimorando suas rotinas de nutrição enteral. Já quanto ao volume, foi considerado adequado o volume infundido $\geq 90 \%$ do volume diário planejado, conforme proposto por Teixeira et al. ${ }^{12}$.

Para a análise das variáveis do estado nutricional foi utilizado o IMC, levando em consideração a idade dos pacientes, sendo que esses foram agrupados em magreza, eutrofia e excesso de peso para análise dos dados. Para os adultos foi utilizada a classificação proposta pela Organização Mundial de Saúde ${ }^{13}$ e para os idosos, a proposta por Lipschitz ${ }^{14}$.

Os resultados foram tabulados no programa Microsoft Excel. Posteriormente foi feita análise estatística no Statistical Package for the Social Sciences (SPSS), versão 20.0. Os dados de idade, gênero, doença(s), início e dias de terapia de nutrição enteral, estado nutricional e volume de dieta infundido/dia foram avaliados descritivamente. Foram realizados cruzamentos relacionando o estado nutricional (magreza, eutrofia e excesso de peso) dos pacientes internados com adequação calórica e proteica $\geq 60 \%$ e também com adequação do volume recebido ( $\geq 90 \%)$. As associações entre essas variáveis foram verificadas através do teste do $\chi^{2}$. As diferenças entre os volumes médios recebidos por cada grupo de estado nutricional foram avaliadas pelo teste ANOVA (análise de variância). O nível de significância utilizado como critério de aceitação ou rejeição nos testes estatísticos foi de $5 \%(\mathrm{p}<0,05)$.

\section{RESULTADOS}

O presente estudo contou com 36 pacientes, com idade média de $54,39 \pm 19,62$ anos, sendo $72,2 \%$ do gênero feminino. O principal motivo de admissão dos pacientes na UTI foi trauma, somando $47,22 \%$ (n=17) dos casos.

As medianas do intervalo entre a internação na UTI e o atendimento ou início da terapia de nutrição enteral, e da permanência nesta foram de 48 horas (24-72) e 168 horas (120-264), respectivamente (percentis 25 e 75 entre parênteses).

Quanto ao estado nutricional dos pacientes na primeira avaliação nutricional durante a internação, levando em conta a classificação do IMC, obteve-se 16,7\% em estado de magreza, 44,44\% em eutrofia e $38,9 \%$ com excesso de peso.

A média de ingestão calórica prescrita para os pacientes foi de $1698,89 \pm 318,35 \mathrm{Kcal} /$ dia. Todos os pacientes em estado de magreza alcançaram $\geq 60 \%$ de adequação das calorias prescritas, enquanto que a maior parte dos que estavam em excesso de peso não atingiram o percentual esperado (Tabela 1 ). O teste do $\chi^{2}$ indicou a existência de associação estatisticamente significante entre as variáveis estado nutricional e adequação calórica $(\mathrm{p}=0,012)$.

A prescrição proteica feita aos pacientes foi em média de $86,56 \pm 23,10 \mathrm{~g} /$ dia. A maioria dos pacientes em estado de magreza atingiu $\geq 60 \%$ de adequação das proteínas prescritas e 33,3\% receberam o volume prescrito. Os pacientes que estavam em excesso de peso não atingiram o previsto (Tabela 2). Novamente, o teste do $\chi^{2}$ indicou a existência de associação estatisticamente significante entre as variáveis estado nutricional e adequação proteica $(\mathrm{p}=0,012)$.

Tabela 1: Pacientes que atingiram $\geq 60 \%$ de adequação das calorias prescritas e administradas durante a internação na unidade de terapia intensiva $(n=36)$ em relação ao estado nutricional $(p=0,012)$

\begin{tabular}{|l|c|c|c|c|c|} 
Classificação & \multicolumn{2}{|c|}{$\begin{array}{c}\text { Atingiram } \\
\mathbf{2 6 0} \%\end{array}$} & \multicolumn{2}{c|}{$\begin{array}{c}\text { Não atingiram } \\
\mathbf{2 6 0 \%}\end{array}$} & \multirow{2}{*}{ Total } \\
\cline { 1 - 5 } & $\mathbf{n}$ & $\%$ & $\mathbf{n}$ & $\%$ & \\
\hline Magreza & 6 & 100 & - & - & 6 \\
\hline Eutrofia & 5 & 31,3 & 11 & 68,8 & 16 \\
\hline Excesso de peso & 5 & 35,7 & 09 & 64,3 & 14 \\
\hline Total & 16 & 44,4 & 20 & 55,5 & 36 \\
\hline
\end{tabular}


A adequação do volume prescrito $(\geq 90 \%)$ relacionado com o administrado da formulação enteral foi atingida por apenas $36,1 \%$ $(\mathrm{n}=13)$ dos pacientes avaliados (Tabela 3). A média do volume recebido pelos pacientes foi de $780,85 \pm 193,12 \mathrm{~mL} / 24 \mathrm{~h}$ durante o período da internação e o volume prescrito para todos os pacientes foi de $1000 \mathrm{~mL} /$ dia. Destaca-se que os pacientes em estado nutricional de magreza receberam o maior volume de dieta $(833,83 \pm 148,46 \mathrm{~mL})$, seguido pelo estado nutricional de excesso de peso $(773,22 \pm 233,88 \mathrm{~mL})$ e eutrofia $(767,67 \pm 175,80 \mathrm{~mL})$. No entanto, essas diferenças não apresentaram significância estatística pelo teste ANOVA ( $\mathrm{p}=0,771$ ).

\section{DISCUSSÃO}

Na presente pesquisa, a média encontrada do intervalo entre a internação na UTU e o início da terapia de nutrição enteral foi de aproximadamente $52 \pm 45$ horas. Já Teixeira et al. ${ }^{12}$ encontraram uma média de 25,3 \pm 20 horas da internação até a introdução da dieta; Cartolano et al. ${ }^{15}$ encontraram um tempo médio de 25,3 e 28,6 horas para o início da terapia de nutrição enteral em estudo comparativo, respectivamente em 2005 e 2008, dados bem mais satisfatórios.

Consensualmente, acredita-se que o quanto antes se inicie o suporte nutricional, melhor. A introdução precoce da dieta entre 24 e 48 horas após entrada na UTI, com o objetivo de atingir o valor energético alvo até as primeiras 48 a 72 horas, pode preservar o estado nutricional com manutenção do peso corporal e da massa muscular e redução do balanço nitrogenado negativo ${ }^{8,16}$.

Um estudo da Sociedade Brasileira de Nutrição Parenteral e Enteral de 1997 observou que aproximadamente 30\% dos pacientes hospitalizados tornavam-se desnutridos nas primeiras

Tabela 2: Pacientes que atingiram $\geq 60 \%$ de adequação das proteínas prescritas e administradas durante a internação na unidade de terapia intensiva $(n=36)$ em relação ao estado nutricional $(p=0,012)$

\begin{tabular}{|c|c|c|c|c|c|}
\hline \multirow[t]{2}{*}{ Classificação } & \multicolumn{2}{|c|}{$\begin{array}{l}\text { Atingiram } \\
\geq 60 \%\end{array}$} & \multicolumn{2}{|c|}{$\begin{array}{c}\text { Não atingiram } \\
\geq 60 \%\end{array}$} & \multirow[t]{2}{*}{ Total } \\
\hline & n & $\%$ & $\mathrm{n}$ & $\%$ & \\
\hline Magreza & 4 & 66,7 & 2 & 33,3 & 6 \\
\hline Eutrofia & 3 & 18,8 & 13 & 81,3 & 16 \\
\hline Excesso de peso & 1 & 7,1 & 13 & 92,9 & 14 \\
\hline Total & 8 & 22,2 & 28 & 77,8 & 36 \\
\hline
\end{tabular}

Tabela 3: Pacientes que atingiram $\geq 90 \%$ de adequação do volume prescrito e administrado durante a internação na unidade de terapia intensiva $(n=36)$ em relação ao estado nutricional $(p=0,896)$

\begin{tabular}{|l|c|c|c|c|c|} 
Classificação & \multicolumn{2}{|c|}{$\begin{array}{c}\text { Receberam } \\
\mathbf{9} \mathbf{9 0 \%}\end{array}$} & \multicolumn{2}{|c|}{$\begin{array}{c}\text { Não receberam } \\
\mathbf{2 9 0 \%}\end{array}$} & \multirow{2}{*}{ Total } \\
\cline { 1 - 5 } & $\mathbf{n}$ & $\%$ & $\mathbf{n}$ & $\%$ & \\
\hline Magreza & 2 & 33,3 & 4 & 66,7 & 6 \\
\hline Eutrofia & 5 & 31,3 & 11 & 68,8 & 16 \\
\hline Excesso de peso & 6 & 42,9 & 8 & 57,1 & 14 \\
\hline Total & 13 & 36,1 & 23 & 63,9 & 36 \\
\hline
\end{tabular}

48 horas de internação. Em 3 a 7 dias esse percentual aumentava em $15 \%$, chegando a $60 \%$ depois de 15 dias de internação ${ }^{17}$.

A nutrição enteral precoce tem sido associada também a menores taxas de complicações infecciosas e menor tempo de permanência na $\mathrm{UTI}^{18}$, bem como à melhora da permeabilidade do trato gastrointestinal ${ }^{4,19}$. Um cuidado proposto pelas diretrizes da $\mathrm{ESPEN}^{8}$ diz respeito aos pacientes críticos poderem estar inicialmente instáveis do ponto de vista metabólico, com alterações hidroeletrolíticas, hiperglicemia de difícil controle, acidose metabólica significativa ou hemodinamicamente muito instáveis. Portanto, clinicamente, é mais sensato retardar o suporte nutricional até que se atinja uma situação metabólica mais estável ${ }^{4}$.

O paciente crítico possui características inerentes, pois se trata de um paciente sujeito a inúmeras alterações dos valores predeterminados para os distintos compartimentos corporais. A resposta ao estresse provoca mudanças nos líquidos intra e extracelulares e no metabolismo ao nível celular, refletindo em medidas antropométricas alteradas, o que dificulta a interpretação dos resultados dos dados coletados e, consequentemente, a avaliação do estado nutricional ${ }^{4,20}$.

A adequada avaliação do estado nutricional pode estimar riscos de mortalidade e morbidade, identificando e individualizando a melhor terapia nutricional. Isso depende da compreensão da fisiopatologia das respostas metabólicas às grandes cirurgias, doenças infecciosas, traumas e eventos similares que tão comumente são rotinas na $\mathrm{UTI}^{20}$.

O IMC é um simples indicador de estado nutricional e, como outros parâmetros de avaliação, tem suas limitações especialmente no paciente crítico. Perdas não intencionais da massa corporal maiores do que $10 \%$ nos últimos 6 meses ou perda mais rápida são prognóticos da evolução clínica de desnutrição, embora possa ser difícil determinar a real perda no paciente crítico. Embora se considere como eutrofia, para os adultos, valores entre $18,5 \mathrm{~kg} / \mathrm{m}^{2}$ e $24,9 \mathrm{~kg} / \mathrm{m}^{2}$, índices inferiores a $20 \mathrm{~kg} / \mathrm{m}^{2}$ devem preocupar, pois são também indicadores de provável desnutrição e podem estar associados ao aumento da mortalidade em diferentes tipos de pacientes ${ }^{21}$.

Verificou-se, neste estudo, que a maioria dos pacientes da UTI estava eutrófico e com excesso de peso no início do acompanhamento nutricional, porém, quanto ao intervalo referente ao recebimento da dieta enteral, da adequação do volume de dieta e da ingestão calórica e proteica, pode-se observar que muitos pacientes não tiveram suas exigências nutricionais atendidas, o que pode conduzir a um aumento do estado nutricional de magreza, já que se trata de pacientes críticos, com todas as suas possíveis intercorrências.

Portanto, os pacientes já em estado nutricional de magreza tiveram suas necessidades nutricionais atingidas, fato que pode ser atribuído a uma atenção maior por parte da equipe de suporte nutricional, já que se trata de pacientes em maior risco de morbidade e mortalidade.

Oliveira et al. ${ }^{22}$, em estudo conduzido em uma UTI de São Paulo em 2008 e 2009, ressaltam a boa adequação entre os valores de 
energia prescrita e administrada, atingindo $88,2 \%$ de adequação. Apontam que o percentual de adequação encontrado reflete a condução de práticas consistentes com o protocolo de infusão de nutrição enteral adotado, definindo medidas para o alcance da meta de $100 \%$ e para o manejo de intercorrências como volume gástrico residual elevado e empenho da EMTN em minimizar tempo de jejum para extubação e procedimentos de rotina.

Teixeira et $a l .^{12}$, analisando a adequação de terapia de nutrição enteral em UTI adulto, encontraram adequação de calorias e proteínas respectivamente, de 74,4 e 71,4\% em relação ao prescrito; no entanto, a adequação de volume administrado em relação ao volume prescrito não alcançou o total planejado. Já Cartolano et al. ${ }^{15}$, levantando dados em uma UTI para melhoria da qualidade da assistência nutricional aos pacientes, apontaram positiva aproximação entre a meta prescrita e os valores de energia e proteína realmente administrados entre os anos de 2005 e 2008. Em 2005, a adequação foi de $74 \%$, tanto em termos de calorias como de proteínas, já em 2008, essa adequação passou para 89\% (meta=90\%).

Aranjues et $a l^{23}$, discutindo a monitoração da terapia de nutrição enteral na UTI como indicador de qualidade, nos anos de 2005 e 2006, revelaram adequação de $74 \%$ em 2005 e $80 \%$ em 2006, em relação ao administrado e o prescrito. E Assis et al. ${ }^{24}$, avaliando as diferenças entre nutrição enteral prescrita e administrada a adultos internados em UTI durante 30 dias no ano de 2009 , concluíram que os pacientes receberam menos volume, calorias e proteínas do que o prescrito. Observaram que $40 \%$ do volume não foram administrados, colaborando para a menor ingestão de calorias e proteínas. Campanella ${ }^{25}$, também em um estudo brasileiro, verificou que apenas $31 \%$ dos pacientes monitorados atingiram a meta calórica proteica determinada.

É importante considerar que, na literatura, os estudos com pacientes críticos apresentam valores semelhantes aos encontrados no presente trabalho, embora alguns, com resultados bem mais satisfatórios, relatem UTI nas quais o monitoramento da qualidade/quantidade da nutrição enteral vem sendo feito já há alguns anos e com grande envolvimento de EMTN.

Estudos demonstram que a terapia de nutrição enteral frequentemente pode resultar em oferta insuficiente de energia e proteínas e que a porcentagem de administração da nutrição enteral em
UTI pode ser variável e atingir de 50 a 100\% do objetivo calórico desejado $^{26}$. Ainda, na prática clínica, pode acontecer que pacientes recebam apenas 50 a $87 \%$ da prescrição dietética ${ }^{27}$.

Muitos fatores concorrem para a dificuldade em se alcançar um aporte nutricional adequado. Embora esse aspecto não tenha sido objetivo neste estudo, todos os autores citados sugerem procedimentos de rotina na UTI como, por exemplo, jejum para exames e procedimentos diagnósticos, rotinas com o paciente (banho, fisioterapia, administração de medicamentos pela sonda, curativos, etc.), procedimentos como extubação orotraquial, repassagem da sonda, traqueostomia, intervenções cirúrgicas e jejum anterior a essas, fatores relacionados à intolerância gastrointestinal (náuseas e vômitos, distensão abdominal, constipação ou diarreia e suas complicações) e instabilidade hemodinâmica como significativos impedimentos de uma adequada nutrição enteral ${ }^{28}$.

Outro aspecto a ser levado em consideração é que hoje sugere-se que não se alimente o paciente acima de suas necessidades energéticas. A hiperalimentação pode ser tão desastrosa quanto a subalimentação. A hiperglicemia pode causar alterações metabólicas graves como a hiperosmolaridade, a diarreia osmótica, desidratação e alterações eletrolíticas, diminuindo as defesas antiinfecciosas ${ }^{4}$. Preconiza-se que a terapia de nutrição enteral excessivamente hipercalórica ou hipocalórica seja evitada em pacientes $\operatorname{críticos}^{22,29}$.

Verificou-se que a maioria dos pacientes estava em estado nutricional de eutrofia ou sobrepeso, de acordo com o IMC. As adequações entre o prescrito e o recebido da dieta enteral com relação ao volume, as calorias e as proteínas precisam ser melhoradas para atender as exigências nutricionais dos pacientes durante a internação na UTI. Destaca-se, positivamente, que os pacientes em estado nutricional de magreza foram os que mais atingiram o aporte calórico, proteico e volume prescritos, de acordo com os critérios propostos no estudo.

A presente pesquisa foi pioneira no hospital onde foi realizado e, com certeza, contribuirá para que estratégias sejam adotadas a fim de atingir maior qualidade na assistência prestada, estabelecendo protocolos de atendimento em terapia de nutrição enteral para melhora desses índices de adequação.

\section{REFERÊNCIAS}

1. Agência Nacional de Vigilância Sanitária (ANVISA). RDC $n^{\circ} 63$, de 6 de julho de 2000. Disponível em: http://www.saude.mg.gov.br/ images/documentos/RDC\%2063\%20NUTRICaO\%20ENTERAL.pdf Acesso em: 23 abr. 2011.

2. Waitzberg DL. Nutrição enteral no doente cirúrgico: avanços e possibilidades. In: Gonçalves EL, Waitzberg DL. Metabolismo na prática cirúrgica. São Paulo: Sarvier; 1993. p. 145-54.

3. Mahan LK, Escott-Stump S. Alimentos, nutrição e dietoterapia. Rio de Janeiro: Elsevier; 2010. p. 506-14
4. Ribeiro PC. Avaliação e suporte nutricional no paciente crítico. In: Schettino G, Cardoso LF, Mattar J. Paciente crítico, diagnóstico e tratamento: Hospital Sírio-Libanês. São Paulo: Manole; 2006. p. 57-70.

5. Correia MI, Waitzberg DL. The impact of malnutrition on morbidity, mortality, length of hospital stay and costs avaluated through a multivariate model analysis. Clin Nutr. 2003;22(3):235-9. http://dx.doi.org/10.1016/S0261-5614(02)00215-7

6. Augusto ALP. Indicações do suporte nutricional. As bases da alimentação enteral. In: Augusto ALP, Alves DC, Mannarino C. Terapia Nutricional. São Paulo: Atheneu; 1995. p. 38-41. 
7. Santana-Cabrera L, O'Shanahan-Navarro G, García-Martul M, Rodríguez AR, Sanchez-Palacios M, Medina EH. Calidad del soporte nutricional artificial em uma unidad de cuidados intensivos. Nutr Hosp. 2006;21(6):661-6.

8. Kreymann KG, Berger MM, Deutz NE, Hiesmayr M, Jolliet $P$, Kazandjiev $G$, et al. ESPEN Guidelines on enteral nutrition: intensive care. Clin Nutr. 2006;25(2):210-23. http://dx.doi.org/10.1016/..clnu.2006.01.021

9. McClave SA, Martindale RG, Vanek VW, McCarthy M, Roberts P. Taylor B, et al. Guidelines for the provision and assessment of nutrition support therapy in the adult critically ill patient: Society Of Critical Care Medicine (SCCM) and American Society for Parenteral and Enteral Nutrition (A.S.P.E.N.). JPEN J Parenter Enteral Nutr. 2009;33(3):277-316. http://dx.doi.org/10.1177/0148607109335234

10. Cerra FB, Benitez MR, Blackbourn GL, Irwin RS, Jeejeebhoy K, Katz DP, et al. Applied nutrition in ICU patients: a consensus treatment of American College of Chest Physicians. Chest. 1997;111(3):769-78 http://dx.doi.org/10.1378/chest.111.3.769

11. CUPPARI, L (Coord.). Guia de nutrição: nutrição clínica no adulto. 2 ed. Barueri: Manole; 2005. 474 p.

12. Teixeira ACC, Caruso L, Soriano FG. Terapia nutricional enteral em unidades de terapia intensiva: infusão versus necessidades. Rev Bras Ter Intensiva. 2006;18(4):331-7. http://dx.doi.org/10.1590/S0103-507X2006000400003

13. World Health Organization (WHO). Obesity: preventing and managing the global epidemic. Geneva: WHO; 1997.

14. Lipschitz DA. Screening for nutritional status in the elderly. Prim Care. 1994;21(1):55-67.

15. Cartolano FC, Caruso L, Soriano FG. Terapia nutricional enteral: aplicacão de indicadores de qualidade. Rev Bras Ter Intensiva. 2009;21(4):376-83 http://dx.doi.org/10.1590/S0103-507X2009000400007

16. Waitzberg DL, Fadul RA, van Aanholt DPJ. Indicações e técnicas de ministração em nutrição enteral In: Waitzberg DL. Nutrição oral, enteral e parenteral na prática clínica. São Paulo: Atheneu; 2000. p. 561-71.

17. Correia MITD, Caiaffa WT, Waitzberg DL. Inquérito brasileiro de avaliação nutricional hospitalar (IBRANUTRI): metodologia do estudo multicênctrico. Rev Bras Nutr Clin. 1998;13(1):30-40.
18. Correia MITD, Echenique M. Custo/benefício da terapia nutricional. In: Waitzberg DL. Nutrição oral, enteral e parenteral na prática clínica. São Paulo: Atheneu; 2000. p. 1635-40.

19. Rocha RGA. Importância da terapia nutricional no paciente grave. In: David CM (Org.). Medicina intensiva. Associação de Medicina Intensiva Brasileira. Rio de Janeiro: Revinter; 2004. p. 980-2.

20. Vasconcelos MIL, Tirapegui J. Aspectos atuais na terapia nutricional de pacientes na unidade de terapia intensiva (UTI). Rev Bras Cienc Farm. 2002;38(1):23-32 http://dx.doi.org/10.1590/S1516-93322002000100003

21. Kamimura MA, Baxmann A, Sampaio L. In: Cuppari L. Guia de nutrição: nutrição clínica do adulto. São Paulo: Manole; 2002. p. $71-109$

22. Oliveira NS, Caruso L, Bergamaschi DP, Cartolano FC, Soriano FG Impacto da adequação da oferta energética sobre a mortalidade em pacientes de UTI recebendo nutrição enteral. Rev Bras Ter Intensiva. 2011;23(2):183-9.

http://dx.doi.org/10.1590/S0103-507X2011000200011

23. Aranjues AL, Teixeira ACC, Caruso L, Soriano FG. Monitoração da terapia nutricional enteral em UTI: indicador de qualidade? Mundo Saúde (Online). 2008;32(1):16-23.

24. Assis MCS, Silva SMR, Leães DM, Novello CL, Silveira CRM, Mello ED, et al. Nutrição enteral: diferenças entre volume, calorias e proteínas precritos e administrados em adultos. Rev Bras Ter Intensiva. 2010;22(4):346-50. http://dx.doi.org/10.1590/S0103-507X2010000400006

25. Campanella LCA, Silveira BM, Rosário Neto O, Silvia AA. Terapia nutricional enteral: a dieta prescrita é realmente infundida? Rev Bras Nutr Clin. 2008;23(1):21-7.

26. Webster NR, Galley HF. Nutrition in the critically ill patient. J R Coll Surg Edinb. 2000;45(6):373-9.

27. Van den Broek PW, Rasmussen-Conrad EL, Naber AH, Wanten GJ. What you think is not what they get: significant discrepancies between prescribed and administered doses of tube feeding. $\mathrm{Br} \mathrm{J}$ Nutr. 2009;101(1):68-71.

http://dx.doi.org/10.1017/S0007114508986852.

28. Vasconcelos MIL. Aspectos práticos na atuação do nutricionista em UTI. Programa Abbott de Especialização em Terapia Intensiva. 1999.

29. Vasconcelos MIL. Nutrição enteral. In: Cuppari L. Guia de nutrição: nutrição clínica do adulto. São Paulo: Manole; 2002. p. 369-90. 